Mater. Res. Soc. Symp. Proc. Vol. 140702012 Materials Research Society

DOI: $10.1557 / 0$ pl.2012.543

\title{
Effect of Dry Oxidation on the Performance of Carbon Nanotube Arrays Electrochemical Capacitors
}

\author{
Adrianus I. Aria ${ }^{1}$ and Morteza Gharib

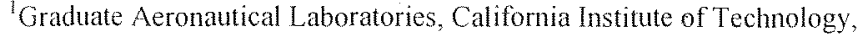 \\ Pasadena, CA 91125 , U.S.A
}

\begin{abstract}
In this study, the effect of dry oxidation on the electrochemical properties of carbon nanotube arrays is investigated. Oxygenated surface functional groups were introduced to the arrays by oxygen plasma treatment, where their surface concentrations were varied by controlling the exposure time. The finding presented herein shows an augmentation of nearly thirty times in term of specific capacitance when the arrays are oxidized. Similar behavior is also observed in the non-aqueous electrolytes where the specific capacitance of the oxidized carbon nanotube arrays is measured more than three times higher than that of the pristine ones. However, overexposure to oxygen plasma treatment reverses this effect. At such high oxidation level, the damage to the graphitic structure becomes more pronounced such that the capacitive behavior of the arrays is overshadowed by their resistive behavior. These findings are important for further development of carbon nanotube based electrochemical capacitors.
\end{abstract}

\section{INTRODUCTION}

In recent years, the performance of electrochemical double-layer capacitors (EDLC) has been increased significantly since the introduction of carbon nanotube (CNT) arrays as their electrodes that replace the standard activated carbon electrodes. Such advancement was possibly achieved because of the inherent physical and electrical properties of the CNT arrays: they have a very large surface area and a very high electrical conductivity [1-3]. Although a lot of works have been done to increase the performance of CNT based EDLC in term of power and energy density using various electrolytes [4-6], the effect of surface chemistry to such performance is yet to be investigated. It was thought that the surface chemistry of CNT electrodes would play a very important role in their electrochemical behavior and performance. We hypothesize that the specific capacitance of pristine CNT electrodes will be much less than that of oxidized ones due to the inherent incompatibility between the non-polar CNT and the polar electrolyte (figure 1).

Current methods for introducing oxygen adsorbates to the CNT originate mainly from wetchemical methods, including the well-established Hummer's method [7]. These methods usually use harsh chemicals, such as strong acids or oxidizing agents, to introduce oxygenated functional groups to the CNT. In contrast, no harsh chemicals are involved in the dry oxidation methods, allowing them to be used safely and conveniently. In this study, the dry oxidation process was done using an oxygen plasma treatment. The concentration of oxygenated functional groups attached to the CNT can be varied by controlling the plasma exposure dose [8]. 
a

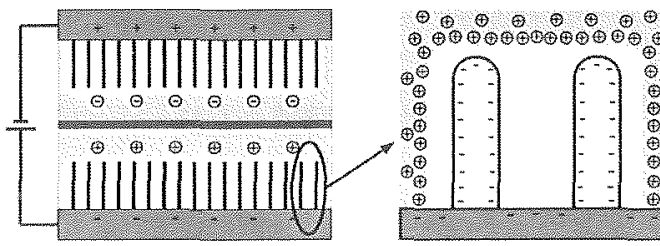

b

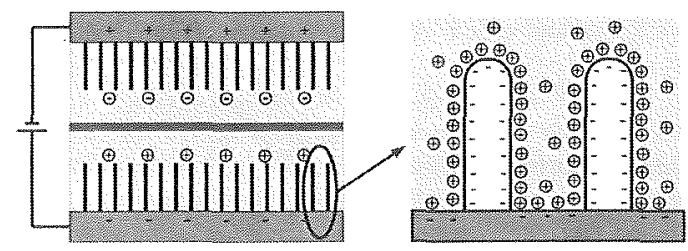

Current collector

CNT array electrodes

Separator

Electrolyte

Figure 1. Schematic drawing of a CNT based EDLC. Typical configuration of such capacitor consists of two symmetric current collectors and CNT electrodes, a non-conducting polymer separator, and an electrolyte. The effective wetted area of the electrodes is influenced by their surface chemistry. Pristine CNT electrodes (a) have a significantly less effective wetted area compared to the oxidized ones (b) due to the inherent incompatibility between the non-polar CNT and the polar electrolyte.

In this work we investigate the effect of dry oxidation, i.e. oxygen plasma treatment, on the electrochemical properties of CNT based EDLC. Here we demonstrate that the specific capacitance of CNT based EDLC is indeed dictated by the surface concentration of oxygenated functional groups. In order to clearly show the effect of oxidation, a smaller potential range was chosen such that the faradaic behavior of the current collector did not dominate the non-faradaic behavior of the CNT electrode. Nevertheless, the result presented herein is comparable to the previously reported study [9].

\section{EXPERIMENT}

Vertically aligned CNT arrays used in this study were grown using chemical vapour deposition (CVD) on catalyst coated silicon substrates with ethylene and hydrogen as the precursor gasses [10]. The length of these arrays was chosen to be $800 \mu \mathrm{m}-1000 \mu \mathrm{m}$. These arrays were then transferred onto copper foil tape and released from their growth substrates. Here, the copper foil tape is used as the current collector of the electrodes. Subsequently, two CNT arrays with copper foil current collector were sandwiched with $25 \mu \mathrm{m}$ thick polyethene separator.

Electrochemical measurements were carried out using potentiostat/galvanostat (BioLogic SP-200) in two different electrolytes: $6 \mathrm{M}$ potassium hydroxide electrolyte (KOH) to simulate aqueous electrolyte, and $1 \mathrm{M}$ of tetraethylammonium tetrafluoroborate $\left(\mathrm{Et}_{4} \mathrm{NBF}_{4}\right)$ in propylene carbonate $(\mathrm{PC})$ to simulate non-aqueous electrolyte. Cyclic voltammetry $(\mathrm{CV})$ and charge- 
discharge cyclic galvanostatic were used to assess the specific capacitance of the CNT electrodes in the abovementioned electrolytes.

a

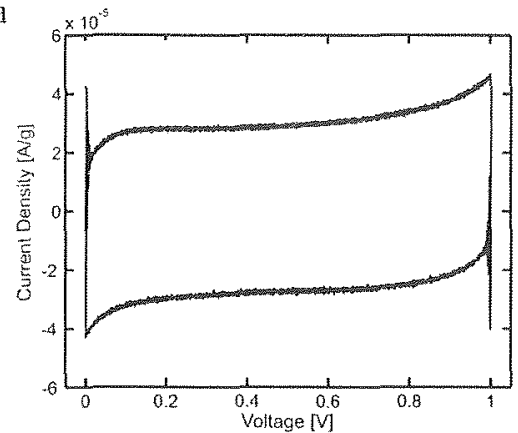

$c$

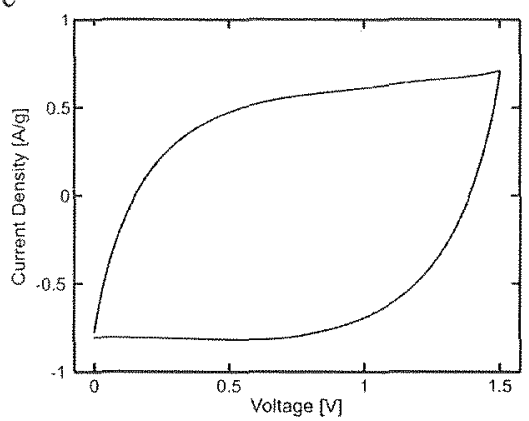

b

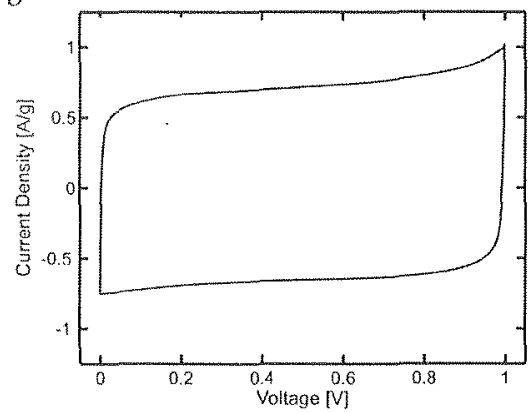

d

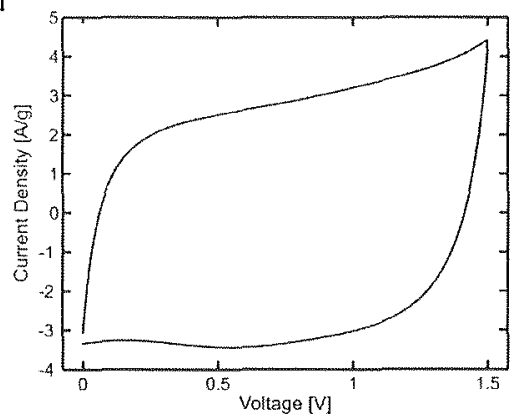

Figure 2. Typical cyclic voltammograms of $\mathrm{CNT}$ based electrochemical capacitor in aqueous and non-aqueous electrolytes. A pristine CNT based capacitor with oxygen/carbon atomic ratio of $-2 \%$ (a) have a significantly less specific capacitance in $6 \mathrm{M} \mathrm{KOH}$ aqueous electrolyte compared to the oxidized one with oxygen/carbon atomic ratio of $\sim 10 \%$ (b). Likewise, a pristine CNT based capacitor with oxygen/carbon atomic ratio of $\sim 3 \%$ (c) have a significantly less specific capacitance in $1 \mathrm{MEt}$ ENBF $4 / \mathrm{PC}$ non-aqueous electrolyte compared to the oxidized one with oxygen/carbon atomic ratio of $\sim 12 \%$ (d).

Oxygenated surface functional groups were introduced to the CNT arrays by oxygen plasma treatment. Such oxidation treatment was performed in a remote RF oxygen plasma cleaner (Tepla M4L) under an oxygen gas flow of $150 \mathrm{sccm}$, a pressure of $500 \mathrm{mtorr}$, and a RF power of 50 watt. The surface concentration of these functional groups was varied by controlling the oxygen plasma exposure time and measured using $x$-ray photoelectron spectroscopy (Surface Science M-Probe XPS). The $x$-ray photoelectron spectroscopy spectral analysis was performed using a Gaussian-Lorentzian curve-fit with Shirley baseline correction. The qualitative defect measurement was conducted using Raman spectroscopy (Renishaw M1000) obtained with excitation energy of $2.41 \mathrm{eV}$. 


\section{RESULTS AND DISCUSSION}

As expected, the capacitive behavior of CNT based electrodes in both aqueous and nonaqueous electrolytes is dictated by their surface chemistry. Since a pristine CNT is inherenty non-polar, the effective wetting area of the pristine CNT electrodes in polar electrolytes is extremely small. Therefore, the specific capacitance of the pristine CNT electrodes is also found to be very low. The cyclic voltammogram of the pristine $\mathrm{CNT}$ electrode in $6 \mathrm{M} \mathrm{KOH}$ (figure $2 \mathrm{a}$ ) shows a good rectangular and symmetric shape over a potential range of $0-1 \mathrm{~V}$. Such featureless voltammogram indicates the absence of redox reactions between the electrodes and the electrolyte. Similarly, the cyclic voltammogram of the oxidized CNT electrode in $6 \mathrm{M} \mathrm{KOH}$ is also leatureless (figure 2b). However, its specific capacitance is measured to be much higher than that of the pristine one. The specific capacitance of the CNT electrodes in $6 \mathrm{M} \mathrm{KOH}$ increases as the increase of oxygen plasma exposure time. In fact, the specific capacitance increases almost thirty times from $\sim \mathrm{F} / \mathrm{g}$ to $-31 \mathrm{~F} / \mathrm{g}$ as the oxygen/carbon atomic ratio of the electrodes increases from $\sim 2 \%$ to $\sim 10 \%$ (figure $3 a$ ).

di

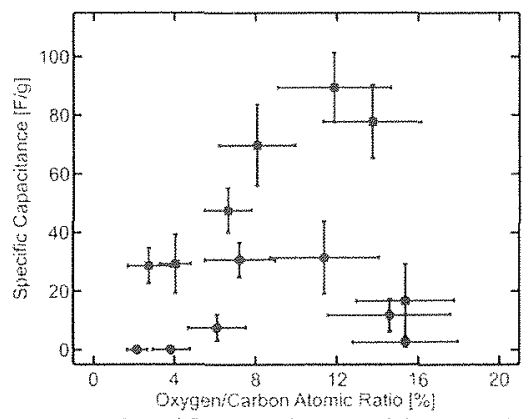

b

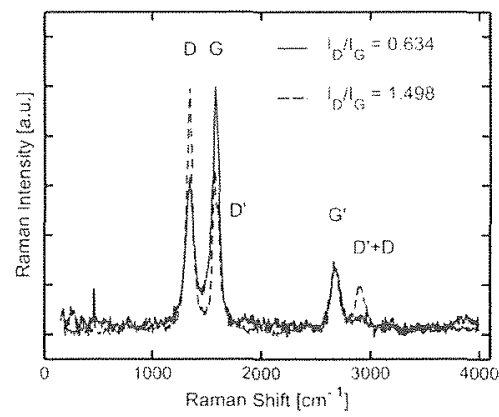

Figure 3. Specific capacitance of the CNT based electrochemical capacitors as a function of their oxygen/carbon atomic ratio (a) in $6 \mathrm{M} \mathrm{KOH} \mathrm{(-)} \mathrm{and} \mathrm{IM} \mathrm{Et4NBF} 4 / P C$ (a) electrolytes. Typical Raman spectra of pristine (oxygen/carbon atomic ratio of $-3 \%$ ) and highly oxidized (oxygen/carbon atomic ratio of $-14 \%$ ) CNT electrodes, with $\mathrm{I}_{\mathrm{D}} / \mathrm{I}_{\mathrm{G}}=-0.6$ for the pristine electrodes (solid line) and $I_{D} / I_{G}=\sim 0.6$ for the oxidized ones (dashed line).

The cyclic voltammogram of the pristine CNT electrode in IM EL4NBF4/PC (figure 2c) shows a good symmetric shape over a potential range of $0-1.5 \mathrm{~V}$. As mentioned earlier, such featureless voltammogram indicates the absence of redox reactions between the electrodes and the electrolyte. Similarly, based on the cyclic voltammogram of the oxidized CNT electrode in IM E14NBF4/PC (figure 2d), its specific capacitance is measured to be much higher than that of the pristine one. The specific capacitance increases almost three times from $\sim 29 \mathrm{~F} / \mathrm{g}$ to $\sim 89 \mathrm{~F} / \mathrm{g}$ as the oxygen/carbon atomic ratio of the electrodes increases from $-3 \%$ to $\sim 12 \%$ (figure $3 \mathrm{a}$ ). This result is comparable to the published data where a specific capacitance of $80 \mathrm{~F} / \mathrm{g}$ can be achieved at oxygen adsorbates concentration of $10.8 \%[9]$. It is worth to note that the ETANBF $4 / P C$ electrolyte itself is capable to deliver a potential window of $-3.5 \mathrm{~V}$. However, a potential window 
of $1.5 \mathrm{~V}$ was chosen because of the faradaic behavior of the current collector at a potential higher than $1.5 \mathrm{~V}$. At such high potential window, the faradaic behavior of the current collector dwarfed the non-faradaic behavior of the carbon nanotube electrode itself, such that a correct measurement of the capacitance could not be performed. Therefore, a potential window of $1.5 \mathrm{~V}$ was chosen in order to probe the effect of oxidation on the capacitance of carbon nanotube electrode without being interfered by the faradaic behavior of the current collector. This faradaic behavior itself seems to be independent to the concentration of oxygen adsorbates introduced to the CNT electrode by plasma treatment.

Although in general the specific capacitance increases as the increase of surface concentration of oxygenated functional groups, which is indicated by the increase of oxygen/carbon atomic ratio, the increase does not really start until the oxygen/carbon atomic ratio reaches $\sim 5 \%$. Above such threshold, the specific capacitance increases monotonically as the increase of oxygen/carbon atomic ratio. However, this effect is reversed once the CNT electrodes undergo a prolonged oxygen plasma exposure time. The specific capacitance plummets significantly once the oxygen/carbon atomic ratio goes beyond $\sim 13 \%$. In fact, the specific capacitance of heavily oxidized CNT electrodes becomes lower than $20 \mathrm{~F} / \mathrm{g}$ at oxygen/carbon atomic ratio goes higher than $14 \%$ for both aqueous and non-aqueous electrolytes.

At such high oxidation level, the graphitic structure of the CNT becomes more defective such that its resistive behavior becomes more pronounced. Such defect can be qualitatively measured from the Raman spectra of the electrodes. The Raman spectra of the pristine CNT electrodes (oxygen to carbon atomic ratio of $\sim 3 \%$ ) show a very low $\mathrm{I}_{\mathrm{D}} / \mathrm{I}_{\mathrm{G}}$ of $\sim 0.6$. In contrast, the over-oxidized CNT electrodes (oxygen to carbon atomic ratio of $\sim 16 \%$ ) show a very high $\mathrm{I}_{\mathrm{D}} / \mathrm{l}_{\mathrm{G}}$ of -1.5 (figure $3 b$ ). Since the disorder-induced $D$ band is related to the non-sp 2 bonding, while the $\mathrm{G}$ band is correlated to the $\mathrm{sp} 2$ lattice symmetry, a considerably high $\mathrm{I}_{\mathrm{D}} / \mathrm{I}_{\mathrm{G}}$ obtained from the over-oxidized CNT electrode suggests that its defect density becomes significantly high such that its pi-bond network is extensively disrupted. Consequently, the electron mobility of such electrode becomes very low as it turns into less conductive and its resistive behavior starts to eclipse its capacitive behavior.

\section{CONCLUSIONS}

In conclusion, the effect of oxidation process on the specific capacitance of CNT capacitors is obvious, where a higher concentration of oxygenated functional groups gives a higher specific capacitance in both aqueous and non-aqueous electrolytes. However, overexposure to oxygen plasma treatment reverses this effect since the damage to the graphitic structure becomes more pronounced such that the capacitive behavior of the arrays is overshadowed by their resistive behavior. In comparison to other reported works, the result presented herein is considered quite low. A better current collector with no noticeable faradaic behavior needs to be used in the future to completely characterize such dry oxidation effect without being limited in a certain potential window.

\section{ACKNOWLEDGMENTS}

This work was supported by The Office of Naval Research under grant number N000 1 4-111-0031 and The Fletcher Jones Foundation under grant number 9900600. The authors gratefully acknowledge the Charyk Laboratory for Bioinspired Design, Kavli Nanoscience Institute (KNI) 
and the Molecular Materials Research Center (MMRC) at the California Institute of Technology for access to fabrication and characterization instruments. The authors also acknowledge Professor George Rossman at the Division of Geological and Planetary Sciences of the California Institute of Technology for access to the Raman spectrometer and Melanie Guittet for her valuable assistance in conducting the experiment.

\section{REFERENCES}

1. E. Frackowiak, K. Metenier, V. Bertagna and F. Beguin, Appl. Phys. Lett. 77, 2421 (2000).

2. J. Barisci, G. Wallace and R. Baughman, J. Electrochem. Soc. 147, 4580-4583 (2000).

3. R. Signorelli, D. C. Ku, J. G. Kassakian and I. E. Schindall, Proc. IEEE 97, 1837-1847 (2009).

4. P. Simon and Y. Gogotsi, Nat. Mater, 7, 845-854 (2008).

5. J. H. Chen, W. Z. Li, D. Z. Wang, S. X. Yang, J. G. Wen and Z. F. Ren, 40, 1193-1197 (2002).

6. J. Fernandez, M. Arulepp, J. Leis, F. Stoeckli and T. Centeno, Electrochim. Acta $\mathbf{5 3}$, $7111-7116(2008)$.

7. W. S. Hummers and R. E. Offeman, J. Am. Chem. Soc. 80, 1339 (1958).

8. T. Xu, J. Yang, J. Liu and Q. Fu, Appl. Surf. Sci. 253, 8945-8951 (2007).

9. H. Pan, J. Y. Li and Y. P. Feng, Nanoscale Res. Lett. 5, 654-668 (2010).

10. A. I. Aria and M. Gharib, Langmuir 27, 9005-9011 (2011). 